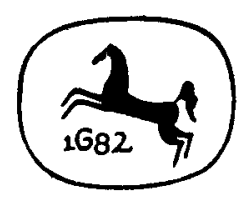

GESCHICHTE DER DEUTSCHEN LITERATUR VON DEN ANFÄNGEN BIS ZUM

ENDE DES SPÄTNITTELALTERS (1490) 
FELIX GENZMER - HELMUT DE BOOR - HUGO KUHN FRIEDRICH RANKE-SIEGFRIED BEYSCHLAG

\section{Geschichte \\ der deutschen Literatur von den Anfängen bis zum Ende des Spätmittelalters (1490)}

J. B. METZLERSCHE VERLAGSBUCHHAN DLUNG STUTTGART MCMLXII 
Aus :

ANNALEN DER DEUTSCHEN LITERATUR

Geschichte der deutschen Literatur von den Anfängen bis zur Gegenwart

Eine Gemeinschaftsarbeit zahlreicher Fachgelehrter

Herausgegeben von Heinz Otto Burger

Zweite Auflage 1962

ISBN 978-3-476-99328-1

ISBN 978-3-476-99327-4 (eBook)

DOI 10.1007/978-3-476-99327-4

(C) 1962 Springer-Verlag GmbH Deutschland

Ursprünglich erschienen bei J. B. Metzlersehe Verlagsbuchhandlung

und Carl Ernst Poeschel Verlag GmbH in Stuttgart 1952/1962 
VORGESCHICHTLICHE UND FRÜHGESCHICHTLICHE ZEIT 2000-770. Von Prof. Dr. Felix Genzmer ( $\dagger$ ), Universität Tübingen $\ldots \ldots \ldots \ldots .1$

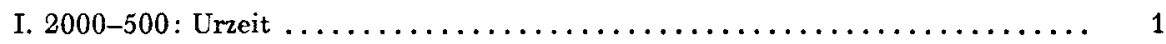

II. 500-200: Entstehung eines besonderen germanischen Stils ............ 3

III. $200 \mathrm{v},-200 \mathrm{n}$. Chr. : Frühgermanischer Stil $\ldots \ldots \ldots \ldots \ldots \ldots \ldots \ldots \ldots \ldots \ldots \ldots \ldots \ldots \ldots \ldots$

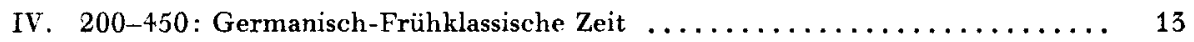

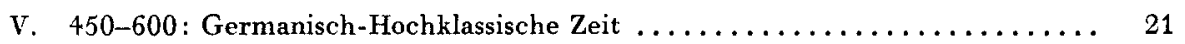

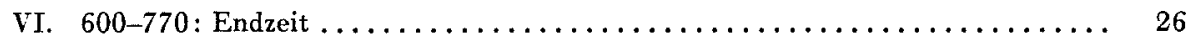

VON DER KAROLINGISCHEN ZUR CLUNIAZENSISCHEN EPOCHE 770-1170. Von Prof. Dr. Helmut de Boor, Freie Universität Berlin ........ $\quad 37$

I. Die karolingische Literatur; Chronologische Gliederung ............ 40

1. 770-790: Vor- und frühkarlische Literatur ................. 40

2. 790-820: Blüte der karlischen Kirchenprosa $\ldots \ldots \ldots \ldots \ldots \ldots \ldots \ldots .43$

5. 820-840: Die hrabanische Periode ....................... 52

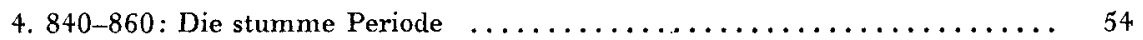

5. 860-880: Die neue Dichtung, Otfried ..................... 56

6. 880-900: Ausbreitung der Reimversdichtung ................ 59

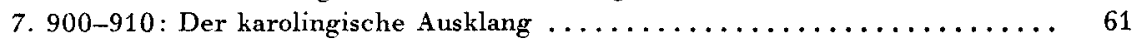

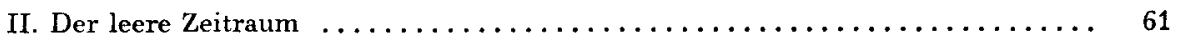

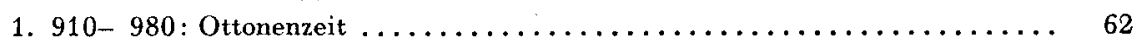

2. 980-1020: Die Zeit Notkers des Deutschen ................... 64

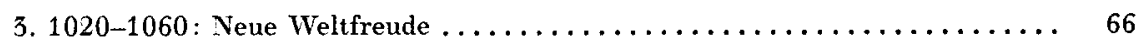

III. Die cluniazensisch-frühmittelhochdeutsche Literatur $\ldots \ldots \ldots \ldots \ldots \ldots \ldots \ldots$

1. 1060-1070: Erstes Wiedererwachen des deutschen Buches .......... 70

2. 1070-1100: Der Einbruch des Kirchenkampfes ................ 73

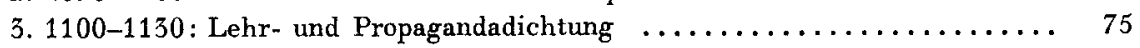

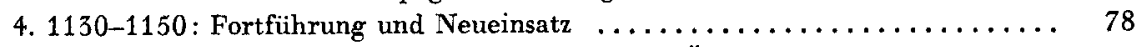

5. 1150-1170: Ausklang der asketischen Dichtung, Öfnung der Welt ...... 84

DIE KLASSIK DES RITTERTUMS IN DER STAUFERZEIT

1170-1230. Von Prof. Dr. Hugo Kuhn, Universität München .............. 99

I. 1170-1180. Der symbolische Naturalismus frühhöfischer Dichtung . . . . . . 102

1. Randgebiete ................................ 102 


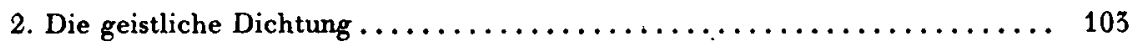

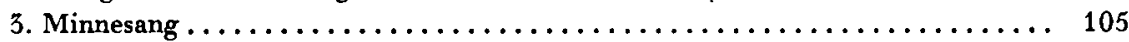

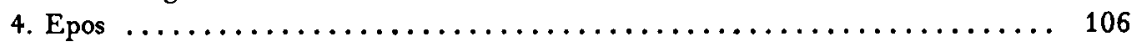

II. 1180-1190. Weltdienst und Gottesdienst als Analogie und Gegensatz ....... 113

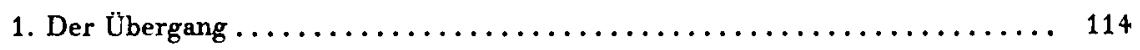

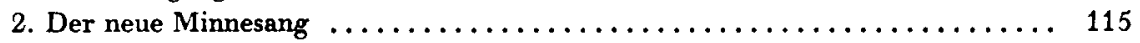

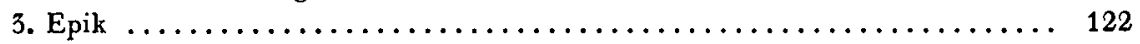

III. 1190-1200. Die Kunstwelt des höfischen Idealismus ............... 127

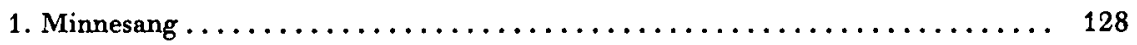

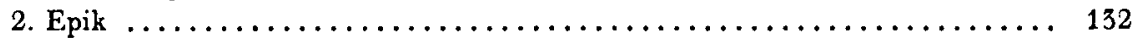

IV. 1200-1210. Dichterischer Weltdienst und Wirklichkeit ............. 136

1. Die politische und die ethische Wirklichkeit in der Lyrik ........... 137

2. Die religiöse Wirklichkeit im Epos ..................... 144

v. 1210-1220. Abschied vom höfischen Idealismus ................. 157

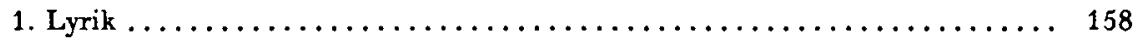

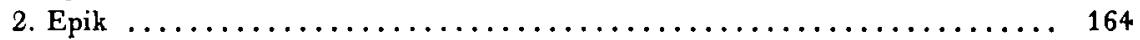

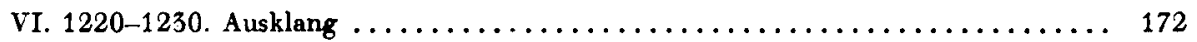

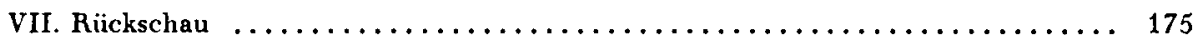

VON DER RITTERLICHEN ZUR BÜRGERLICHEN DICHTUNG

1230-1430. Von Prof. Dr. Friedrich Ranke (t), Universität Basel ......... 179

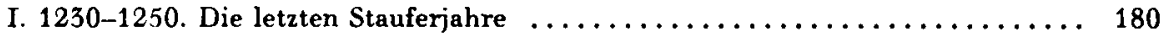

1. Epigonenleistung in Ritterroman und höfischer Legende $\ldots \ldots \ldots \ldots \ldots \ldots 180$

2. Späthöfischer Heldenroman ........................... 181

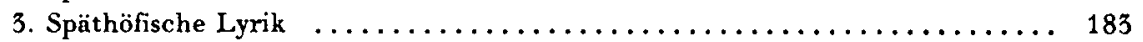

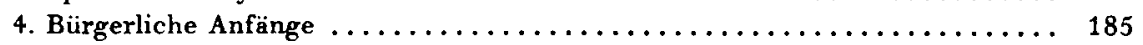

5. Verfrühte Erzählprosa und erstes Schauspiel $\ldots \ldots \ldots \ldots \ldots \ldots \ldots \ldots \ldots 186$

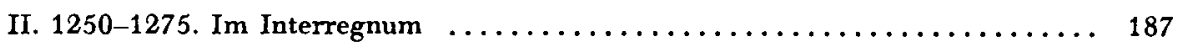

1. Ausbreitung der Ritterepik . ......................... 187

2. Entartung der Heldenepik $\ldots \ldots \ldots \ldots \ldots \ldots \ldots \ldots \ldots \ldots \ldots \ldots \ldots \ldots \ldots$

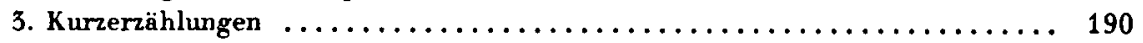

4. Minnesang und seine Parodie; Sangsprüche der Fahrenden ............ 191

5. Franziskanerpredigt; Mystik in Prosa und Vers ................ 192

III. 1275-1300. Unter Rudolf von Habsburg und Adolf von Nassau ............ 194

1. "Gotik « in der Dichtung; Uberschreiten und Ausschreiten der Wirklichkeit . 194

2. Minnesang der Stadtbürger und Fürsten; Balladen ............... 198

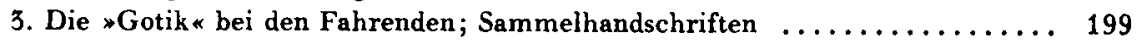

4. Ende der Heldenepik; Geschichtsdichtung ................... 201

5. Zeitkritik und Lehrdichtung $\ldots \ldots \ldots \ldots \ldots \ldots \ldots \ldots \ldots \ldots \ldots, 202$

6. Aufstieg der geistlichen Dichtung $\ldots \ldots \ldots \ldots \ldots \ldots \ldots \ldots \ldots \ldots \ldots \ldots \ldots$

7. Geistliches Schauspiel; Prosa ......................... 204 
IV. 1300-1325. Zwiespalt in Staat und Kirche. Einssein mit Gott ........... 205

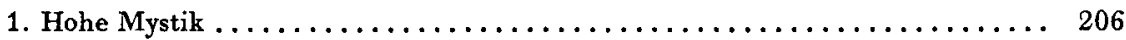

2. Die Hochflut der geistlichen Dichtung $\ldots \ldots \ldots \ldots \ldots \ldots \ldots \ldots \ldots \ldots, 207$

3. Verfall des Minnesangs und letzte Ritterepen $\ldots \ldots \ldots \ldots \ldots \ldots \ldots \ldots . \ldots \ldots$

4. Geschichtsdichtung und Kurzerzählungen $\ldots \ldots \ldots \ldots \ldots \ldots \ldots \ldots \ldots .211$

V. 1325-1350. Unter Ludwig dem Bayern ...................... 212

1. Ausbreitung und Verflachung der Mystik .................... 212

2. Geistliches Spiel und Deutschordensdichtung $\ldots \ldots \ldots \ldots \ldots \ldots \ldots \ldots 216$

3. Weltliche Lehrdichtung geistlicher Verfasser $\ldots \ldots \ldots \ldots \ldots \ldots \ldots \ldots .218$

4. Verfall des Ritterepos ............................... 218

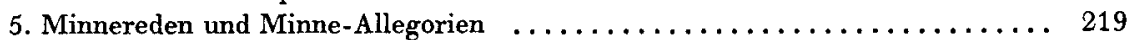

6. Ehrenreden und historisch-politische Lieder $\ldots \ldots \ldots \ldots \ldots \ldots \ldots \ldots 222$

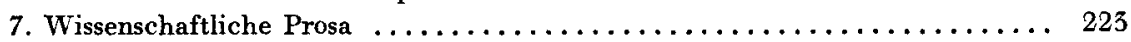

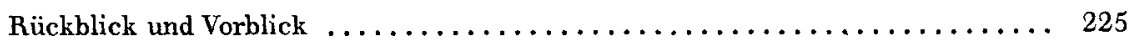

VI. 1350-1370: Zur Zeit Karls IV. ........................ 227

1. Das Neue als Form: Johann $v$. Neumarkt $\ldots \ldots \ldots \ldots \ldots \ldots \ldots \ldots \ldots . \ldots 227$

2. Meistersingerische Gelehrsamkeit ..................... 227

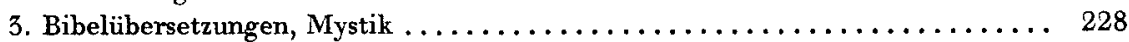

4. Zeitkritik, Preis- und Lehrdichtung, lehrhafte Kurzerzählung ......... 230

5. Lied und Drama in den Städten, erste weltliche Spiele ............ 231

VII. 1370-1400: Um den Gottesfreund vom Oberland und den Mönch von Salzburg • 233

1. Hochflut geistlicher Prosa .......................... 233

2. Die Welt der Gegensätze: Geistliches Spiel, Kirchenlied und weltliche Lyrik . 235

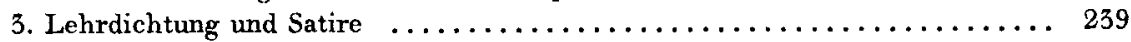

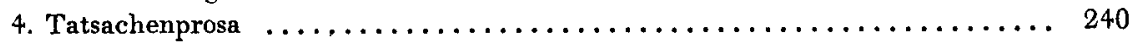

VIII. 1400-1410: Ein Jahrzehnt der großen Dichtungen ................ 241

1. Johann von Tepl und Oswald von Wolkenstein; Der Ring und die Theologia

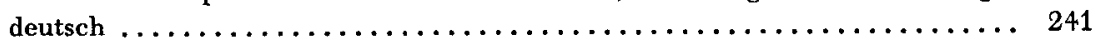

2. Heinrich Kaufringer und Hans der Büheler $\ldots \ldots \ldots \ldots \ldots \ldots \ldots \ldots . \ldots . \ldots . \ldots . \ldots 245$

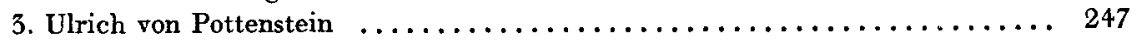

IX. 1410-1430: Konstanzer Konzil und Hussitenkriege ............... 247

1. Oswalds reife Lyrik . ........................... 247

2. Zeitklage, Satire, Lehrdichtung (Muskatplüt, Vintler, Rothe) ......... 248

3. Weltliche Spiele vom Tanawäschel und von Neidhart $\ldots \ldots \ldots \ldots \ldots \ldots \ldots 250$

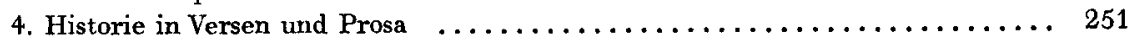

5. Religiöse Lehre und Erbauung in Prosa und Vers ............... 252

STÄDTE, HÖFE, GELEHRTE 1430-1490. Von Prof. Dr. Siegfried Beyschlag, Universität

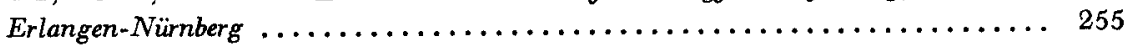

I. 1430-1450: Die Grundlage; Spiel und Literatur der städtischen Gemeinschaften 256

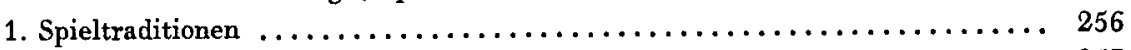

2. Geistliches Schrifttum und Historisches Lied ................ 257

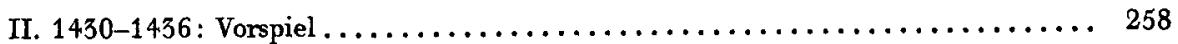

1. 1430-1434: Die Anfänge von Hartlieb und GroB $\ldots \ldots \ldots \ldots \ldots \ldots \ldots . \ldots \ldots$

2. 1434-1437: Philosophische und praktische Welterfassung ........... 259 


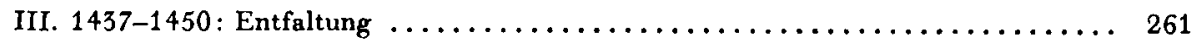

1. $1437:$ Der Prosaroman ............................... 261

2. 1437-1440: Erfassung der geistigen Substanz $\ldots \ldots \ldots \ldots \ldots \ldots \ldots \ldots . \ldots \ldots 2$

3. 1441-1449: Dichtung und Schrifttum der Städte und Höfe ........... 263

IV. 1450-1460: Scheitelhöhe des Jahrhunderts; Neue Richtungen .......... 265

1. 1450: Die Stadt ............................... 265

2. 1450-1456: Spiegelung neuhöfischer Ritterkultur; Fortgang des lateinsprachi-

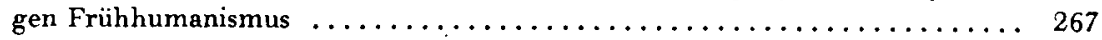

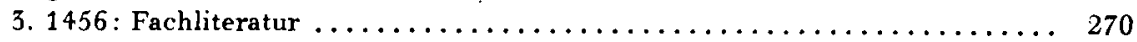

4. Vor und nach 1456: Politische Dichtung; Rosenplüt............ 270

5. Um 1460 : Erste Höhe des Frühhumanismus und letzte Werke des Kusaners .. 272

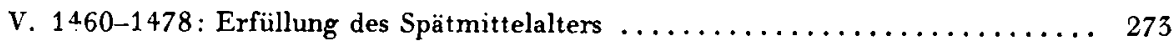

1. 1461-1465: Ritterromantik; die neue Spielform ............... 274

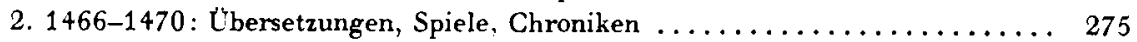

3. 1470-1475: Erfüllung alter und neuer Formen.............. 276

4. 1476-1478: Neue Welle mittelhochdeutscher Renaissance; Historisches Lied . 279

5. Um 1478: Letzte Höhe des Frühhumanismus ............... 280

V. 1479-1490: Ausklang und neue Ansätze ..................... 281

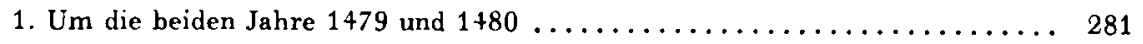

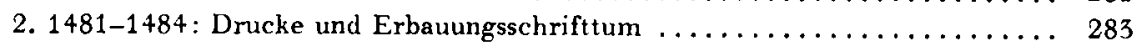

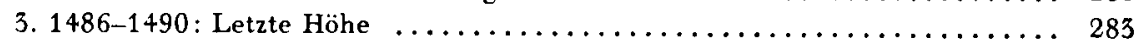

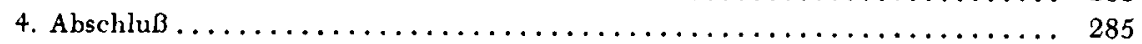

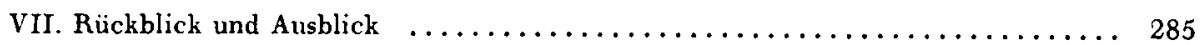

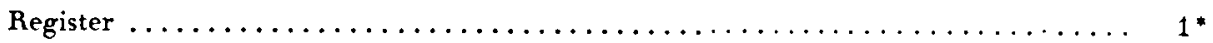

